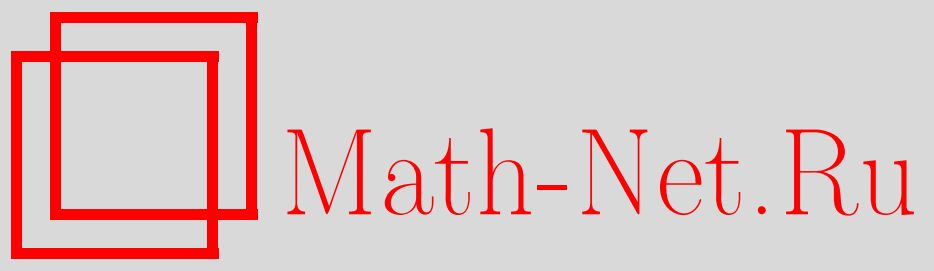

А. М. Шапиро, Три реализации квантовой аффинной алгебры $U_{q}\left(A_{2}^{(2)}\right), T M \Phi, 2010$, том 165 , номер 2, 217-232 DOI: https://doi.org/10.4213/tmf6572

Использование Общероссийского математического портала Math-Net.Ru подразумевает, что вы прочитали и согласны с пользовательским соглашением http://www . mathnet.ru/rus/agreement

Параметры загрузки:

IP : 3.82 .47 .9

26 апреля 2023 г., 18:22:18

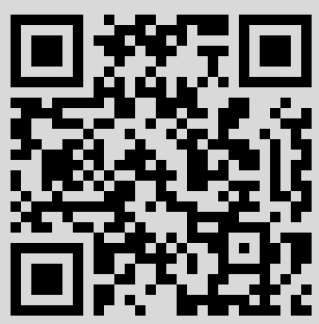




\title{
ТРИ РЕАЛИЗАЦИИ КВАНТОВОЙ АФФИННОЙ АЛГЕБРЫ $U_{q}\left(A_{2}^{(2)}\right)$
}

\begin{abstract}
Установлены явные изоморфизмы между тремя реализациями квантовой скрученной аффинной алгебры $U_{q}\left(A_{2}^{(2)}\right)$ : токовой реализацией Дринфельда, реализацией Шевалле и так называемой $R L L$-реализацией, предложенной $\mathrm{Pe}$ шетихиным, Тахтаджяном и Фаддеевым.
\end{abstract}

Ключевые слова: квантовые аффинные алгебры, $R L L$-реализация.

\section{1. ВВЕДЕНИЕ}

Существуют лишь две квантовые аффинные алгебры ранга $2: U_{q}\left(\widehat{\mathfrak{s l}}_{2}\right)$ и $U_{q}\left(A_{2}^{(2)}\right)$. Алгебра $U_{q}\left(\widehat{\mathfrak{s l}}_{2}\right)$ и ее теория представлений хорошо изучены. Ситуация с алгеброй $U_{q}\left(A_{2}^{(2)}\right)$ несколько иная, несмотря на то что и алгебра сама по себе, и ее теория представлений интересны как с точки зрения математики, так и с точки зрения математической физики.

Впервые алгебра $U_{q}\left(A_{2}^{(2)}\right)$ возникла в физических работах. Так, фундаментальное представление ее $\mathcal{R}$-матрицы было получено в работе [1] в качестве $\mathcal{R}$-матрицы квантовой версии модели Шабата-Михайлова, также известной как модель Изергина-Корепина. Позже это же представление было получено в работе [2] вместе с фундаментальными представлениями $\mathcal{R}$-матриц прочих не исключительных аффинных алгебр. Позднее в работе [3] техника анзаца Бете была распространена на алгебру $U_{q}\left(A_{2}^{(2)}\right)$. Наконец, в работе [4] были развиты идеи термодинамического анзаца Бете для алгебры $U_{q}\left(A_{2}^{(2)}\right)$ и выписаны некоторые конечномерные представления.

Алгебра $U_{q}\left(A_{2}^{(2)}\right)$ также изучалась и с алгебраической точки зрения. В работе [5] был построен базис Картана-Вейля для адгебры $U_{q}\left(A_{2}^{(2)}\right)$ и универсальная $\mathcal{R}$-матрица была выписана через бесконечные произведения элементов этого базиса. Классификация конечномерных представлений алгебры $U_{q}\left(A_{2}^{(2)}\right)$ была получена в работе [6] в терминах полиномов Дринфельда. Позднее была получена [7] интегральная формула для универсальной $\mathcal{R}$-матрицы алгебры $U_{q}\left(A_{2}^{(2)}\right)$, при

* Институт теоретической и экспериментальной физики, Москва, Россия. E-mail: alexander.m.shapiro@gmail.com 
этом $U_{q}\left(A_{2}^{(2)}\right)$ рассматривалась как топологическая алгебра Хопфа с коумножением Дринфельда. В той же работе соотношения Серра были переписаны в терминах нулей и полюсов произведений токов Дринфельда. Наконец, факторизованная $\mathcal{R}$-матрица алгебры $U_{q}\left(A_{2}^{(2)}\right)$ была выписана в интегральном виде в статье [8], где $U_{q}\left(A_{2}^{(2)}\right)$ рассматривалась как алгебра со стандартной структурой алгебры Хопфа.

Квантовые аффинные алгебры допускают три различные реализации с разными структурами алгебр Хопфа. Первая из них - стандартная реализация - задается при помощи образующих Шевалле и соотношений, определяемых соответствующей матрицей Картана. Стандартная реализация содержит малое число образующих, однако, к сожалению, крайне неудобна для использования в приложениях. Вторая реализация - новая реализация Дринфельда - впервые описана в работе [9] при помощи производящих функций (токов Дринфельда) и соотношений на них. Реализация Дринфельда позволяет использовать методы комплексного анализа при изучении алгебры. Более того, она позволяет описать все конечномерные представления квантовой аффинной алгебры. Наконец, третья реализация - $R L L-$ реализация - основана на подходе Решетихина, Тахтаджяна, Фаддеева и Семенова-Тянь-Шанского, в котором образующие собраны в $L$-операторы, удовлетворяющие известному уравнению Янга-Бакстера (см. [10], [11]). Простота коумножения в $R L L$-реализации позволяет строить новые представления как тензорные произведения уже известных. По этой причине именно $R L L$-реализация широко используется в физических моделях.

Считается общеизвестным, что описанные реализации квантовых аффинных алгебр изоморфны, несмотря на то что точные доказательства вряд ли существуют для каких-либо алгебр помимо $\mathfrak{s l}_{n}$-серии. Для алгебры $U_{q}\left(\widehat{\mathfrak{s l}}_{n}\right)$ изоморфизм между стандартной реализацией и реализацией Дринфельда был описан в работе [12], а связь между реализацией Дринфельда и $R L L$-реализацией была установлена в [13].

В случае квантовых скрученных аффинных алгебр $R L L$-реализация требует дополнительных соотношений. Хотя считается, что три реализации остаются изоморфными и в случае скрученных алгебр, пока что нет полного понимания, как в точности должны выглядеть изоморфизмы. Изоморфизм между стандартной реализацией и реализацией Дринфельда алгебры $U_{q}\left(A_{2}^{(2)}\right)$ был установлен в работе [8], а в работе [14] был частично установлен изоморфизм между $R L L$-реализацией и реализацией Дринфельда. Более полную библиографию можно найти в работе [15].

В настоящей работе дается полное описание трех реализаций алгебры $U_{q}\left(A_{2}^{(2)}\right)$ (без градуирующего элемента и с нулевым центральным зарядом), изоморфизмов между ними и связей между тремя структурами алгебр Хопфа. Особое внимание уделено следующему факту: каждая реализация обладает "минимальным" (или почти минимальным в случае скрученных алгебр) набором образующих и расширенным набором образующих. Для этих наборов, по всей видимости, должен существовать аналог теоремы Пуанкаре-Биркгофа-Витта. В стандартной реализации такими наборами являются соответственно образующие Шевалле и базис КартанаВейля. В реализации Дринфельда эти функции выполняют токи Дринфельда и так называемые "сложные токи" (см. [16]). Наконец, в $R L L$-реализации гауссовы 
координаты, находящиеся непосредственно над или под диагональю, образуют минимальный набор, а все гауссовы координаты дают расширенный набор. Заметим, что эти расширенные наборы играют ключевую роль при вычислении универсальной весовой функции. В настоящей работе устанавливается связь между проекциями сложных токов и гауссовыми координатами для алгебры $U_{q}\left(A_{2}^{(2)}\right)$ в духе того, как это было проделано для $U_{q}\left(\widehat{\mathfrak{g l}}_{n}\right)$ в работе [17].

Статья построена следующим образом: в разделе 2 даются описания трех реализаций, в разделе 3 устанавливаются изоморфизмы между ними и приводятся доказательства этих изоморфизмов, и, наконец, в разделе 4 записываются элементы из расширенного набора гауссовых координат через сложные токи.

\section{2. РЕАЛИЗАЦИИ АЛГЕБРЫ $U_{Q}\left(A_{2}^{(2)}\right)$}

Для простоты изложения мы рассматриваем алгебру $U_{q}\left(A_{2}^{(2)}\right)$ без градуирующего элемента и с нулевым центральным зарядом. Все утверждения остаются верными и для алгебры с градуирующим элементом и произвольным центральным зарядом, но формулы становятся более громоздкими.

2.1. Реализация Дринфельда. Пусть $\mathcal{A}_{\mathrm{D}}$ - ассоциативная алгебра, порожденная элементами

$$
e_{n}, f_{n}, \quad n \in \mathbb{Z}, \quad a_{n}, \quad n \in \mathbb{Z} \backslash\{0\}, \quad \text { и } \quad k^{ \pm 1},
$$

отвечающими определенным соотношениям. Соотношения заданы на производящих функциях (токах) в виде равенств формальных степенных рядов

$$
e(z)=\sum_{k \in \mathbb{Z}} e_{k} z^{-k}, \quad f(z)=\sum_{k \in \mathbb{Z}} f_{k} z^{-k}, \quad K^{ \pm}(z)=k^{ \pm 1} \exp \left( \pm\left(q-q^{-1}\right) \sum_{n>0} a_{ \pm n} z^{\mp n}\right)
$$

следующим образом:

$$
\begin{aligned}
\left(z-q^{2} w\right)(q z+w) e(z) e(w) & =\left(q^{2} z-w\right)(z+q w) e(w) e(z), \\
\left(q^{2} z-w\right)(z+q w) f(z) f(w) & =\left(z-q^{2} w\right)(q z+w) f(w) f(z), \\
K^{+}(z) e(w) K^{+}(z)^{-1} & =\alpha\left(\frac{w}{z}\right) e(w), \\
K^{+}(z) f(w) K^{+}(z)^{-1} & =\alpha\left(\frac{w}{z}\right)^{-1} f(w), \\
K^{-}(z) e(w) K^{-}(z)^{-1} & =\alpha\left(\frac{z}{w}\right)^{-1} e(w), \\
K^{-}(z) f(w) K^{-}(z)^{-1} & =\alpha\left(\frac{z}{w}\right) f(w), \\
K^{ \pm}(z) K^{ \pm}(w) & =K^{ \pm}(w) K^{ \pm}(z), \\
K^{-}(z) K^{+}(w) & =K^{+}(w) K^{-}(z), \\
e(z) f(w)-f(w) e(z) & =\frac{1}{q-q^{-1}}\left(\delta\left(\frac{z}{w}\right) K^{+}(w)-\delta\left(\frac{z}{w}\right) K^{-}(z)\right),
\end{aligned}
$$


где

$$
\alpha(x)=\frac{\left(q^{2}-x\right)\left(q^{-1}+x\right)}{\left(1-q^{2} x\right)\left(1+q^{-1} x\right)},
$$

а $\delta(z / w)$ - формальный ряд Лорана, заданный равенством

$$
\delta\left(\frac{z}{w}\right)=\sum_{n \in \mathbb{Z}}\left(\frac{z}{w}\right)^{n} .
$$

Производящие функции $e(z), f(z)$ также удовлетворяют кубическим соотношениям Серра (см. [9]):

$$
\begin{aligned}
\operatorname{Sym}_{z_{1}, z_{2}, z_{3}}\left(q^{-3} z_{1}-\left(q^{-2}+q^{-1}\right) z_{2}+z_{3}\right) e\left(z_{1}\right) e\left(z_{2}\right) e\left(z_{3}\right) & =0, \\
\operatorname{Sym}_{z_{1}, z_{2}, z_{3}}\left(q^{-3} z_{1}^{-1}-\left(q^{-2}+q^{-1}\right) z_{2}^{-1}+z_{3}^{-1}\right) f\left(z_{1}\right) f\left(z_{2}\right) f\left(z_{3}\right) & =0 \\
\operatorname{Sym}_{z_{1}, z_{2}, z_{3}}\left(q^{3} z_{1}^{-1}-\left(q^{2}+q\right) z_{2}^{-1}+z_{3}^{-1}\right) e\left(z_{1}\right) e\left(z_{2}\right) e\left(z_{3}\right) & =0 \\
\operatorname{Sym}_{z_{1}, z_{2}, z_{3}}\left(q^{3} z_{1}-\left(q^{2}+q\right) z_{2}+z_{3}\right) f\left(z_{1}\right) f\left(z_{2}\right) f\left(z_{3}\right) & =0 .
\end{aligned}
$$

Алгебра Хопфа на $\mathcal{A}_{\mathrm{D}}$ может быть задана так:

$$
\begin{aligned}
\Delta_{\mathrm{D}}(e(z)) & =e(z) \otimes 1+K^{-}(z) \otimes e(z), \\
\Delta_{\mathrm{D}}(f(z)) & =1 \otimes f(z)+f(z) \otimes K^{+}(z), \\
\Delta_{\mathrm{D}}\left(K^{ \pm}(z)\right) & =K^{ \pm}(z) \otimes K^{ \pm}(z), \\
S_{\mathrm{D}}(e(z)) & =-\left(K^{-}(z)\right)^{-1} e(z), \\
S_{\mathrm{D}}(f(z)) & =-f(z)\left(K^{+}(z)\right)^{-1}, \\
S_{\mathrm{D}}\left(K^{ \pm}(z)\right) & =\left(K^{ \pm}(z)\right)^{-1}, \\
\varepsilon_{\mathrm{D}}(e(z)) & =0, \\
\varepsilon_{\mathrm{D}}(f(z)) & =0, \\
\varepsilon_{\mathrm{D}}\left(K^{ \pm}(z)\right) & =1,
\end{aligned}
$$

где $\Delta_{\mathrm{D}}, \varepsilon_{\mathrm{D}}$ и $S_{\mathrm{D}}$ - соответственно коумножение, коединица и антипод. Мы будем называть $\Delta_{\mathrm{D}}$ коумножением Дринфельда. Здесь мы должны отметить, что $\mathcal{A}_{\mathrm{D}}-$ топологическая биалгебра, и $\Delta_{\mathrm{D}}$ является отображением из алгебры $\mathcal{A}_{\mathrm{D}}$ в топологическое расширение ее тензорного квадрата (см. раздел 2 в работе [18]).

2.2. Реализация Шевалле. Другая реализация алгебры $U_{q}\left(A_{2}^{(2)}\right)$ задается при помощи образующих Шевалле. Пусть $\mathcal{A}_{\mathrm{Ch}}$ - ассоциативная алгебра, порожденная элементами $e_{ \pm \alpha}, e_{ \pm(\delta-2 \alpha)}, k_{\alpha}^{ \pm 1}, k_{\delta-2 \alpha}^{ \pm 1}$, удовлетворяющими соотношениям

$$
\begin{aligned}
& k_{\alpha} e_{ \pm \alpha} k_{\alpha}^{-1}=q^{ \pm 1} e_{ \pm \alpha}, \quad k_{\alpha} e_{ \pm(\delta-2 \alpha)} k_{\alpha}^{-1}=q^{\mp 2} e_{ \pm(\delta-2 \alpha)}, \\
& k_{\delta-2 \alpha} e_{ \pm \alpha} k_{\delta-2 \alpha}^{-1}=q^{\mp 2} e_{ \pm \alpha}, \quad k_{\delta-2 \alpha} e_{ \pm(\delta-2 \alpha)} k_{\delta-2 \alpha}^{-1}=q^{ \pm 4} e_{ \pm(\delta-2 \alpha)} \text {, } \\
& k_{\alpha}^{2} k_{\delta-2 \alpha}=1, \quad\left[e_{ \pm \alpha}, e_{\mp(\delta-2 \alpha)}\right]=0, \\
& {\left[e_{\alpha}, e_{-\alpha}\right]=\frac{k_{\alpha}-k_{\alpha}^{-1}}{q-q^{-1}}, \quad\left[e_{\delta-2 \alpha}, e_{-(\delta-2 \alpha)}\right]=\frac{k_{\delta-2 \alpha}-k_{\delta-2 \alpha}^{-1}}{q-q^{-1}},} \\
& \left(\operatorname{ad}_{q} e_{ \pm \alpha}\right)^{5} e_{ \pm(\delta-2 \alpha)}=0, \quad\left(\operatorname{ad}_{q} e_{ \pm(\delta-2 \alpha)}\right)^{2} e_{ \pm \alpha}=0
\end{aligned}
$$


где

$$
\begin{aligned}
\left(\operatorname{ad}_{q} e_{ \pm \alpha}\right)(x) & =e_{ \pm \alpha} x-k_{\alpha}^{ \pm 1} x k_{\alpha}^{\mp 1} e_{ \pm \alpha} \\
\left(\operatorname{ad}_{q} e_{ \pm(\delta-2 \alpha)}\right)(x) & =e_{ \pm(\delta-2 \alpha)} x-k_{\delta-2 \alpha}^{ \pm 1} x k_{\delta-2 \alpha}^{\mp 1} e_{ \pm(\delta-2 \alpha)} .
\end{aligned}
$$

Структура алгебры Хопфа, ассоциированная с данной реализацией, может быть определена следующим образом:

$$
\begin{gathered}
\Delta\left(e_{\alpha}\right)=e_{\alpha} \otimes 1+k_{\alpha} \otimes e_{\alpha}, \quad \Delta\left(e_{\delta-2 \alpha}\right)=e_{\delta-2 \alpha} \otimes 1+k_{\delta-2 \alpha} \otimes e_{\delta-2 \alpha}, \\
\Delta\left(e_{-\alpha}\right)=1 \otimes e_{-\alpha}+e_{-\alpha} \otimes k_{\alpha}^{-1}, \quad \Delta\left(e_{-(\delta-2 \alpha)}\right)=1 \otimes e_{-(\delta-2 \alpha)}+e_{-(\delta-2 \alpha)} \otimes k_{\delta-2 \alpha}^{-1}, \\
\Delta\left(k_{\alpha}\right)=k_{\alpha} \otimes k_{\alpha}, \quad \Delta\left(k_{\delta-2 \alpha}\right)=k_{\delta-2 \alpha} \otimes k_{\delta-2 \alpha}, \\
\varepsilon\left(e_{ \pm \alpha}\right)=0, \quad \varepsilon\left(e_{ \pm(\delta-2 \alpha)}\right)=0, \\
\varepsilon\left(k_{\alpha}^{ \pm 1}\right)=1, \quad \varepsilon\left(k_{\delta-2 \alpha}^{ \pm 1}\right)=1, \\
S\left(e_{\alpha}\right)=-k_{\alpha}^{-1} e_{\alpha}, \quad S\left(e_{\delta-2 \alpha}\right)=-k_{\delta-2 \alpha}^{-1} e_{\delta-2 \alpha}, \\
S\left(e_{-\alpha}\right)=-e_{-\alpha} k_{\alpha}, \quad S\left(e_{-(\delta-2 \alpha)}\right)=-e_{-(\delta-2 \alpha)} k_{\delta-2 \alpha}, \\
S\left(k_{\alpha}^{ \pm 1}\right)=k_{\alpha}^{\mp 1}, \quad S\left(k_{\delta-2 \alpha}^{ \pm 1}\right)=k_{\delta-2 \alpha}^{\mp 1},
\end{gathered}
$$

где $\Delta, \varepsilon$ и $S$ - соответственно коумножение, коединица и антипод. Мы будем называть $\Delta$ стандартным коумножением. Мы также будем рассматривать противоположное коумножение

$$
\Delta^{\mathrm{op}}=\sigma \circ \Delta, \quad \sigma(u \otimes v)=v \otimes u .
$$

Теперь нам потребуется конструкция базиса Картана-Вейля, построенная для $U_{q}\left(A_{2}^{(2)}\right)$ в работе [5] $]^{1)}$. Скрученная аффинная алгебра $A_{2}^{(2)}$ обладает системой корней $\Delta=\Delta_{+} \cup \Delta_{-}$, где

$$
\Delta_{+}=\alpha \cup\{n \delta, \pm \alpha+n \delta, \pm 2 \alpha+(2 n-1) \delta \mid n \in \mathbb{N}\}, \quad \Delta_{-}=-\Delta_{+} .
$$

Корни $\gamma \in \Delta_{+}$называются положительными, корни $\gamma \in\{n \delta, n \in \mathbb{Z}\}-$ мнимыми, а корни $\gamma \in \Delta \backslash\{n \delta, n \in \mathbb{Z}\}-$ действителъными. Определим скалярное произведение на $\Delta$ :

$$
(\alpha, \alpha)=1, \quad(\alpha, \delta)=(\delta, \delta)=0
$$

Теперь, пусть

$$
q_{\gamma}=q^{(\gamma, \gamma)}, \quad(a)_{q}=\frac{q^{a}-1}{q-1}, \quad(n)_{q} !=(1)_{q} \cdots(n)_{q}
$$

и

$$
\exp _{q}(x)=\sum_{n \geqslant 0} \frac{x^{n}}{(n)_{q} !}=1+x+\frac{x^{2}}{(2)_{q} !}+\cdots .
$$

Зафиксируем нормальное упорядочение на $\Delta$ :

$$
\alpha, 2 \alpha+\delta, \alpha+\delta, 2 \alpha+3 \delta, \alpha+2 \delta, \ldots, \delta, 2 \delta, 3 \delta, \ldots, 2 \delta-\alpha, 3 \delta-2 \alpha, \delta-\alpha, \delta-2 \alpha .
$$

\footnotetext{
1) В нашей конструкции, в отличие от описаной в [5], в построении базиса Картана-Вейля используются $q$-коммутаторы вместо $q^{-1}$-коммутаторов.
} 
В соответствии с конструкцией базиса Картана-Вейля, описанной в работе [5], положим

$$
\begin{gathered}
e_{\delta-\alpha}=a\left[e_{\alpha}, e_{\delta-2 \alpha}\right]_{q^{-1}}, \quad e_{\delta}^{\prime}=b\left[e_{\alpha}, e_{\delta-\alpha}\right]_{q^{-1}}, \quad e_{\alpha+n \delta}=b\left[e_{\alpha+(n-1) \delta}, e_{\delta}^{\prime}\right]_{q^{-1}}, \\
e_{\delta-\alpha+n \delta}=b\left[e_{\delta}^{\prime}, e_{\delta-\alpha+(n-1) \delta}\right]_{q^{-1}}, \quad e_{n \delta}^{\prime}=b\left[e_{\alpha+(n-1) \delta}, e_{\delta-\alpha}\right]_{q^{-1}}, \\
e_{2 \alpha+(2 n+1) \delta}=a\left[e_{\alpha+n \delta}, e_{\alpha+(n+1) \delta}\right]_{q^{-1}}, \quad e_{\delta-2 \alpha+2 n \delta}=a\left[e_{\delta-\alpha+n \delta}, e_{\delta-\alpha+(n-1) \delta}\right]_{q^{-1}},
\end{gathered}
$$
где

$$
a=\frac{1}{\sqrt{q+q^{-1}}}, \quad b=\frac{1}{\sqrt{q+1+q^{-1}}},
$$

и для всех $\beta, \gamma \in \Delta$ выполняется

$$
\left[e_{\beta}, e_{\gamma}\right]_{q^{-1}}=e_{\beta} e_{\gamma}-q^{-(\beta, \gamma)} e_{\gamma} e_{\beta} .
$$

Наконец, определим мнимые корни $e_{n \delta}$ через полиномы Шура:

$$
e_{n \delta}^{\prime}=\sum_{p_{1}+2 p_{2}+\cdots+n p_{n}=n} \frac{\left(\left(q-q^{-1}\right) b^{-1}\right)^{\sum p_{i}-1}}{p_{1} ! \ldots p_{n} !} e_{\delta}^{p_{1}} e_{2 \delta}^{p_{2}} \ldots e_{n \delta}^{p_{n}} .
$$

Мономы, являющиеся произведением элементов $e_{n \delta}, e_{n \delta \pm \alpha}$ и $k_{\alpha}^{ \pm 1}$ образуют линейный базис алгебры $\mathcal{A}_{\mathrm{Ch}}$.

2.3. $R L L$-реализация. Пусть

$$
R(x)=\left(\begin{array}{lllllllll}
1 & 0 & 0 & 0 & 0 & 0 & 0 & 0 & 0 \\
0 & a & 0 & d & 0 & 0 & 0 & 0 & 0 \\
0 & 0 & b & 0 & e & 0 & p & 0 & 0 \\
0 & f & 0 & a & 0 & 0 & 0 & 0 & 0 \\
0 & 0 & g & 0 & c & 0 & e & 0 & 0 \\
0 & 0 & 0 & 0 & 0 & a & 0 & d & 0 \\
0 & 0 & q & 0 & g & 0 & b & 0 & 0 \\
0 & 0 & 0 & 0 & 0 & f & 0 & a & 0 \\
0 & 0 & 0 & 0 & 0 & 0 & 0 & 0 & 1
\end{array}\right),
$$

где

$$
\begin{aligned}
& a=\frac{q(x-1)}{q^{2} x-1}, \quad b=\frac{q^{2}(q x+1)(x-1)}{\left(q^{2} x-1\right)\left(q^{3} x+1\right)}, \quad c=\frac{q(x-1)}{q^{2} x-1}+\frac{\left(q^{2}-1\right)\left(q^{3}+1\right) x}{\left(q^{2} x-1\right)\left(q^{3} x+1\right)}, \\
& d=\frac{q^{2}-1}{q^{2} x-1}, \quad e=\frac{\left(q^{2}-1\right)(x-1) q^{1 / 2}}{\left(q^{2} x-1\right)\left(q^{3} x+1\right)}, \quad p=\frac{\left(q^{2}-1\right)\left(q^{3} x+q x-q+1\right)}{\left(q^{2} x-1\right)\left(q^{3} x+1\right)} \\
& f=\frac{\left(q^{2}-1\right) x}{q^{2} x-1}, \quad g=\frac{\left(1-q^{2}\right) x(x-1) q^{5 / 2}}{\left(q^{2} x-1\right)\left(q^{3} x+1\right)}, \quad q=\frac{\left(q^{2}-1\right) x\left(q^{3} x-q^{2} x+q^{2}+1\right)}{\left(q^{2} x-1\right)\left(q^{3} x+1\right)} .
\end{aligned}
$$

Рассмотрим производящие функции

$$
\begin{array}{ll}
l_{i j}^{ \pm}(z)=\sum_{n=0}^{\infty} l_{i j}^{ \pm}[ \pm n] z^{\mp n}, & 1 \leqslant i, j \leqslant 3, \\
l_{i j}^{+}[0]=l_{j i}^{-}[0]=0, & 1 \leqslant i<j \leqslant 3,
\end{array}
$$


и сдвинутые производящие функции

$$
\tilde{l}_{i j}^{ \pm}(z)=q^{(i-j) / 2} l_{4-j, 4-i}^{ \pm}\left(-q^{-3} z\right) .
$$

Определим $L$-операторы $L^{ \pm}(z)$ и их сдвиги $\tilde{L}^{ \pm}(z)$ следующим образом:

$$
L^{ \pm}(z)=\left(l_{i j}^{ \pm}(z)\right)_{i, j=1}^{3}, \quad \tilde{L}^{ \pm}(z)=\left(\tilde{l}_{i j}^{ \pm}(z)\right)_{i, j=1}^{3} .
$$

Наконец, напомним определение $q$-детерминанта:

$$
\operatorname{det}_{q}\left(L^{ \pm}(z)\right)=\sum_{\tau \in \mathfrak{S}_{3}}(-q)^{\operatorname{sgn} \tau} l_{1 \tau(i)}^{ \pm}(z) l_{2 \tau(2)}^{ \pm}\left(q^{2} z\right) l_{3 \tau(3)}^{ \pm}\left(q^{4} z\right)
$$

где $\mathfrak{S}_{n}$ - группа перестановок множества из $n$ элементов.

Определим ассоциативную алгебру $\mathcal{A}_{R}$ с образующими $l_{i j}^{ \pm}[ \pm n], n \in \mathbb{N}, 1 \leqslant i, j \leqslant 3$, и $l_{i j}^{+}[0], l_{j i}^{-}[0]$ при $1 \leqslant j \leqslant i \leqslant 3$, удовлетворяющими следующим соотношениям:

$$
\begin{gathered}
\operatorname{det}_{q}\left(L^{ \pm}(z)\right)=1, \\
L^{ \pm}(z) \tilde{L}^{ \pm}(z)=I_{3}, \\
l_{i i}^{+}[0] l_{i i}^{-}[0]=l_{i i}^{-}[0] l_{i i}^{+}[0]=1, \\
R\left(\frac{z}{w}\right) L_{1}^{ \pm}(z) L_{2}^{ \pm}(w)=L_{2}^{ \pm}(w) L_{1}^{ \pm}(z) R\left(\frac{z}{w}\right), \\
R\left(\frac{z}{w}\right) L_{1}^{+}(z) L_{2}^{-}(w)=L_{2}^{-}(w) L_{1}^{+}(z) R\left(\frac{z}{w}\right),
\end{gathered}
$$

где $L_{1}(z)=L(z) \otimes I_{3}, L_{2}(z)=I_{3} \otimes L(z)$, а $I_{n}$ обозначает единичную $(n \times n)$-матрицу.

Структура алгебры Хопфа может быть задана на $\mathcal{A}_{R}$ таким образом:

$$
\Delta_{R}\left(l_{i j}^{ \pm}\right)=\sum_{k=1}^{3} l_{i k}^{ \pm} \otimes l_{k j}^{ \pm}, \quad S_{R}\left(L^{ \pm}\right)=\left(L^{ \pm}\right)^{-1}, \quad \varepsilon_{R}\left(L^{ \pm}\right)=I_{3},
$$

где $\Delta_{R}, \varepsilon_{R}$ и $S_{R}$ - соответственно коумножение, коединица и антипод.

$L$-операторы допускают гауссово разложение

$$
L^{ \pm}(z)=\left(\begin{array}{ccc}
1 & f_{1}^{ \pm}(z) & f_{13}^{ \pm}(z) \\
0 & 1 & f_{2}^{ \pm}(z) \\
0 & 0 & 1
\end{array}\right)\left(\begin{array}{ccc}
k_{1}^{ \pm}(z) & 0 & 0 \\
0 & k_{2}^{ \pm}(z) & 0 \\
0 & 0 & k_{3}^{ \pm}(z)
\end{array}\right)\left(\begin{array}{ccc}
1 & 0 & 0 \\
e_{1}^{ \pm}(z) & 1 & 0 \\
e_{31}^{ \pm}(z) & e_{2}^{ \pm}(z) & 1
\end{array}\right)
$$

где

$$
\begin{array}{rlrl}
e_{i}^{ \pm}(z) & =\sum_{n \geqslant 0} e_{i}^{ \pm}[n] z^{\mp n}, & i=1,2, & e_{31}^{ \pm}(z)=\sum_{n \geqslant 0} e_{31}^{ \pm}[n] z^{\mp n}, \\
f_{i}^{ \pm}(z)=\sum_{n \geqslant 0} f_{i}^{ \pm}[n] z^{\mp n}, & i=1,2, & f_{13}^{ \pm}(z)=\sum_{n \geqslant 0} f_{13}^{ \pm}[n] z^{\mp n}, \\
k_{i}^{ \pm}(z)=\sum_{n \geqslant 0} k_{i}^{ \pm}[n] z^{\mp n}, & i=1,2,3, &
\end{array}
$$


и

$$
e_{1}^{-}[0]=e_{2}^{-}[0]=e_{31}^{-}[0]=0, \quad f_{1}^{+}[0]=f_{2}^{+}[0]=f_{13}^{+}[0]=0 .
$$

Таким образом, мы определили гауссовы образующие

$$
f_{1}^{ \pm}[n], f_{2}^{ \pm}[n], f_{13}^{ \pm}[n], \quad e_{1}^{ \pm}[n], e_{2}^{ \pm}[n], e_{31}^{ \pm}[n], \quad k_{1}^{ \pm}[n], k_{2}^{ \pm}[n], k_{3}^{ \pm}[n], \quad n \geqslant 0,
$$

удовлетворяющие соотношениям (4)-(7). Здесь $f_{1}^{ \pm}[n], f_{2}^{ \pm}[n], e_{1}^{ \pm}[n], e_{2}^{ \pm}[n]$ принадлежат "минимальному" набору образующих, а $f_{13}^{ \pm}[n]$ и $e_{31}^{ \pm}[n]-$ расширенному.

\section{3. СВЯЗИ МЕЖДУ РЕАЛИЗАЦИЯМИ}

3.1. Универсальная $\mathcal{R}$-матрица. Напомним, что универсальной $\mathcal{R}$-матрицей квазитреугольной алгебры Хопфа $\mathcal{A}$ называется элемент $\mathcal{R} \in \mathcal{A} \otimes \mathcal{A}$, удовлетворяющий соотношениям

$$
\Delta^{\mathrm{op}}(x)=\mathcal{R} \Delta(x) \mathcal{R}^{-1}
$$

для любого $x \in \mathcal{A}$, и

$$
(\Delta \otimes \mathrm{id}) \mathcal{R}=\mathcal{R}_{13} \mathcal{R}_{23}, \quad(\mathrm{id} \otimes \Delta) \mathcal{R}=\mathcal{R}_{13} \mathcal{R}_{12},
$$

где

$$
\mathcal{R}_{12}=\mathcal{R} \otimes 1, \quad \mathcal{R}_{23}=1 \otimes \mathcal{R}, \quad \mathcal{R}_{13}=(\sigma \otimes \text { id })\left(\mathcal{R}_{23}\right)
$$

являются элементами тензорного куба алгебры $\mathcal{A} \otimes \mathcal{A} \otimes \mathcal{A}$.

Теперь зафиксируем представление алгебры $\pi_{z}$, определяемое на образующих Шевалле следующим образом:

$$
\begin{array}{rlrl}
\pi_{z}\left(e_{\alpha)}=\right. & \left(\begin{array}{ccc}
0 & q^{1 / 4} & 0 \\
0 & 0 & -q^{-1 / 4} \\
0 & 0 & 0
\end{array}\right), & \pi_{z}\left(e_{\delta-2 \alpha}\right) & =\left(\begin{array}{ccc}
0 & 0 & 0 \\
0 & 0 & 0 \\
\sqrt{q+q^{-1}} & 0 & 0
\end{array}\right) z \\
\pi_{z}\left(e_{-\alpha}\right) & =\left(\begin{array}{ccc}
0 & 0 & 0 \\
q^{-1 / 4} & 0 & 0 \\
0 & -q^{1 / 4} & 0
\end{array}\right), & \pi_{z}\left(e_{-\delta+2 \alpha)}\right. & =\left(\begin{array}{ccc}
0 & 0 & \sqrt{q+q^{-1}} \\
0 & 0 & 0 \\
0 & 0 & 0
\end{array}\right) z^{-1}, \\
\pi_{z}\left(k_{\alpha)}=\left(\begin{array}{ccc}
q & 0 & 0 \\
0 & 1 & 0 \\
0 & 0 & q^{-1}
\end{array}\right),\right. & \pi_{z}\left(k_{\delta-2 \alpha)}=\left(\begin{array}{ccc}
q^{-2} & 0 & 0 \\
0 & 1 & 0 \\
0 & 0 & q^{2}
\end{array}\right) .\right.
\end{array}
$$

После того как представление выбрано, мы получаем $R$-матрицу ${ }^{2)}$

$$
R\left(\frac{z}{w}\right)=\left(\pi_{z} \otimes \pi_{w}\right) \mathcal{R}
$$

непосредственно из определения $\mathcal{R}$, применяя $\pi_{z} \otimes \pi_{w}$ к соотношению $(9)$ и подставляя образующие Шевалле вместо $x$. Условие $(9)$ определяет $R(x)$ с точностью до мультипликативной константы.

\footnotetext{
2) Нормировка базиса представления выбрана таким образом, что $R$-матрица $R(x)$ симметрична относительно побочной диагонали. $R$-матрица в этом базисе совпадает с полученной в работе [2].
} 
3.2. Реализации Шевалле и Дринфельда. Следуя [5], мы представляем универсальную $\mathcal{R}$-матрицу алгебры $\mathcal{A}_{\mathrm{Ch}}$ в следующей форме ${ }^{3)}$ :

$$
\begin{array}{ll}
\mathcal{R}=\mathcal{R}_{-} \mathcal{K}^{-h \otimes h} \mathcal{R}_{+}, & \mathcal{R}_{-}=\left(\prod_{\gamma<\delta}^{\rightarrow} \exp _{q_{\gamma}}\left(\left(q^{-1}-q\right) e_{\gamma} \otimes e_{-\gamma}\right)\right), \\
\mathcal{K}=\exp \left(-\sum_{n>0} S_{n}\right), & \mathcal{R}_{+}=\left(\prod_{\gamma>\delta}^{\leftarrow} \exp _{q_{\gamma}}\left(\left(q^{-1}-q\right) e_{\gamma} k_{\alpha}^{-(\alpha, \gamma)} \otimes k_{\alpha}^{(\alpha, \gamma)} e_{-\gamma}\right)\right),
\end{array}
$$

где $\gamma$ пробегает по всем положительным корням, $h$ определяется через $q^{ \pm h}=k^{ \pm 1}$, а $S_{n}$ задается формулой

$$
S_{n}=\frac{n\left(q-q^{-1}\right)^{2}\left(q+1+q^{-1}\right)\left(e_{n \delta} \otimes e_{-n \delta}\right)}{\left(q^{n}-q^{-n}\right)\left(q^{n}+(-1)^{n+1}+q^{-n}\right)} .
$$

Теперь, наконец, мы можем найти скалярный множитель для $R(x)$. Применяя $\pi_{z} \otimes \pi_{w}$ к $\mathcal{K} \cdot q^{-h \otimes h}$, мы получаем скаляр

$$
q^{-1} \exp \left(-\sum_{n>0} \frac{\left(q^{n}-q^{-n}\right) x^{n}}{\left(q^{n}+(-1)^{n+1}+q^{-n}\right) n}\right) .
$$

Таким образом, $R(x)$ совпадает с матрицей (3), умноженной на скаляр (12).

ЗАмЕчАниЕ 1. Скаляр (12) разлагается в произведение

$$
q^{-1} \prod_{k=0}^{\infty}\left(\frac{\left(1+q^{3 k+2} x\right)\left(1-q^{3 k+3} x\right)}{\left(1+q^{3 k} x\right)\left(1-q^{3 k+1} x\right)}\right)^{(-1)^{k}}
$$

в области $|q|<1$ и в произведение

$$
q^{-1} \prod_{k=0}^{\infty}\left(\frac{\left(1+q^{-3 k} x\right)\left(1-q^{-(3 k+1)} x\right)}{\left(1+q^{-(3 k+2)} x\right)\left(1-q^{-(3 k+3)} x\right)}\right)^{(-1)^{k}}
$$

в области $|q|>1$.

Теорема 1. Изоморбизм ассоииативных алгебр $\mathcal{A}_{\mathrm{Ch}}$ и $\mathcal{A}_{\mathrm{D}}$ может быть задан бормулами

$$
\begin{aligned}
k_{\delta-2 \alpha} & \mapsto k^{-2}, & k_{\alpha} & \mapsto k, \\
e_{\delta-2 \alpha} & \mapsto a\left(q f_{1} f_{0}-f_{0} f_{1}\right) k^{-2}, & e_{\alpha} & \mapsto e_{0}, \\
e_{-\delta+2 \alpha} & \mapsto a k^{2}\left(q^{-1} e_{0} e_{-1}-e_{-1} e_{0}\right), & e_{-\alpha} & \mapsto f_{0},
\end{aligned}
$$

где а определено после (1).

${ }^{3)}$ Формула для $\mathcal{R}$-матрицы отличается от приведенной в работе [5], поскольку в построении базиса Картана-Вейля $q$-коммутаторы заменены на $q^{-1}$-коммутаторы. Более того, формула, приведенная здесь, отличается и от приведенной в [8]. $\mathcal{R}$-матрица в [8] является обратной к той, что выписана здесь.

2 Теоретическая и математическая физика, т. 165, № 2, 2010 г. 
ДокАзАтЕльство. Непосредственная проверка и индуктивное построение базиса Картана-Вейля показывают, что отображение (13) является сюръективным гомоморфизмом. Более точно, любая образующая Дринфельда может быть получена следующим образом:

$$
\begin{array}{rlrlrl}
e_{\alpha+n \delta} & \mapsto e_{n}, \quad n \geqslant 0, & -k_{\alpha}^{-1} e_{\alpha-n \delta} & \mapsto e_{-n}, & & n>0, \\
e_{-\alpha-n \delta} & \mapsto f_{-n}, \quad n \geqslant 0, \quad-e_{-\alpha+n \delta} k_{\alpha} & \mapsto f_{n}, & & n>0, \\
k_{\alpha} & \mapsto k, & -b^{-1} e_{n \delta} & \mapsto a_{n}, & & n \in \mathbb{Z} \backslash\{0\},
\end{array}
$$

Обращая направления всех стрелок в отображении (14), мы получаем отображение, обратное к (13). Наконец, отображение (14) с обращенными стрелками также является эпиморфизмом, откуда и следует утверждение теоремы.

ЗАмечАниЕ 2. Отображение, описанное выше, является изоморфизмом ассоциативных алгебр. Оно сохраняет коединицу, но не сохраняет коумножения $\Delta$ и $\Delta_{\mathrm{D}}$.

Следующее предложение связывает стандартное коумножение и коумножение Дринфельда.

ПрЕДЛОЖЕНИЕ 1. Тензор $\mathcal{R}_{-}$является кочиклом по отношению $\kappa \Delta_{\mathrm{D}}$, так что для любого $x \in \mathcal{A}_{\mathrm{Ch}}$

$$
\Delta(x)=\left(\mathcal{R}_{-}\right)^{-1} \Delta_{\mathrm{D}}(x) \mathcal{R}_{-} .
$$

Доказательство дано в работе [8]. Также там можно найти выражение для тензора $\mathcal{R}_{-}$в терминах образующих Дринфельда.

3.3. От реализации Шевалле к $R L L$-реализации. Универсальная $R$-матрица любой квазитреугольной алгебры Хопфа удовлетворяет квантовому уравнению Янга-Бакстера (см. [9])

$$
\mathcal{R}_{12} \mathcal{R}_{13} \mathcal{R}_{23}=\mathcal{R}_{23} \mathcal{R}_{13} \mathcal{R}_{12}
$$

Теорема 2. Изоморфизм ассоииативных алгебр $\mathcal{A}_{R}$ u $\mathcal{A}_{\mathrm{Ch}}$ может быть задан следующими отображениями:

$$
L^{-}(z) \mapsto\left(\pi_{z} \otimes \mathrm{id}\right) \mathcal{R}, \quad L^{+}(z) \mapsto\left(\pi_{z} \otimes \mathrm{id}\right) \mathcal{R}_{21}^{-1}
$$

ДоказАтельство. Применяя $\pi_{z} \otimes \pi_{w} \otimes \mathrm{id}, \pi_{z} \otimes \mathrm{id} \otimes \pi_{w}$ и $\mathrm{id} \otimes \pi_{z} \otimes \pi_{w}$ к соотношению (15), мы выводим коммутационные соотношения (6) и (7). Используя формулы (11) и (16), мы получаем гауссово разложение (8).

Матрица $R\left(-q^{3}\right)$ имеет одномерное ядро, порожденное вектором

$$
v=q^{-1 / 2} v_{1} \otimes v_{3}+v_{2} \otimes v_{2}+q^{1 / 2} v_{3} \otimes v_{1}
$$

где $\left\{v_{1}, v_{2}, v_{3}\right\}$ - базис представления $\pi_{z}$. Вектор $v$ является собственным вектором оператора $L_{1}(z) L_{2}\left(-q^{-3} z\right)$. Пусть $\lambda(z)$ - собственное значение, отвечающее вектору $v$. Оператор $\left(\pi_{z} \otimes \pi_{-q^{-3} z}\right) \Delta(x)$ обращается в нуль на $v$ для любого $x \in \mathcal{A}_{\mathrm{Ch}}$ (последнее достаточно проверить только для образующих Шевалле). Применяя 
$\pi_{z} \otimes \pi_{-q^{-3} z} \otimes$ id к первому уравнению в $(10)$, мы получаем $\lambda(z)=1$. Таким образом, оператор $L_{1}(z) L_{2}\left(-q^{-3} z\right)$ сохраняет $v$, что эквивалентно соотношению (5):

$$
\left(\begin{array}{lll}
l_{11}(z) & l_{12}(z) & l_{13}(z) \\
l_{21}(z) & l_{22}(z) & l_{23}(z) \\
l_{31}(z) & l_{32}(z) & l_{33}(z)
\end{array}\right)\left(\begin{array}{ccc}
l_{33}\left(-q^{-3} z\right) & q^{-1 / 2} l_{23}\left(-q^{-3} z\right) & q^{-1} l_{13}\left(-q^{-3} z\right) \\
q^{1 / 2} l_{32}\left(-q^{-3} z\right) & l_{22}\left(-q^{-3} z\right) & q^{-1 / 2} l_{12}\left(-q^{-3} z\right) \\
q l_{31}\left(-q^{-3} z\right) & q^{1 / 2} l_{21}\left(-q^{-3} z\right) & l_{11}\left(-q^{-3} z\right)
\end{array}\right)=I_{3} .
$$

Далее можно проверить, что

$$
\operatorname{det}_{q}\left(L^{ \pm}(z)\right)=k_{1}^{ \pm}(z) k_{2}^{ \pm}\left(q^{-2} z\right) k_{3}^{ \pm}\left(q^{-4} z\right) .
$$

Следуя построению базиса Картана-Вейля, мы получаем

$$
\pi_{z}\left(e_{ \pm n \delta}\right)=b \frac{q^{n}-q^{-n}}{n\left(q-q^{-1}\right)}\left(\begin{array}{ccc}
-z^{ \pm n} & 0 & 0 \\
0 & \left(q^{2} z\right)^{ \pm n}-(-q z)^{ \pm n} & 0 \\
0 & 0 & \left(-q^{3} z\right)^{ \pm n}
\end{array}\right), \quad n>0 .
$$

Применяя $\pi_{z}$ к первому или второму тензорному сомножителю $\mathcal{K}$, имеем

$$
\begin{aligned}
& k_{1}^{ \pm}(z)=k_{3}^{ \pm}\left(-q^{-3} z\right)^{-1}, \\
& k_{2}^{ \pm}(z)=k_{3}^{ \pm}\left(-q^{-1} z\right) k_{3}^{ \pm}\left(q^{-2} z\right)^{-1},
\end{aligned}
$$

откуда и следует искомое соотношение (4).

Так как все образующие Шевалле лежат в образе отображения (16), мы получили эпиморфизм из $\mathcal{A}_{R}$ на $\mathcal{A}_{\mathrm{Ch}}$ (и, как следствие, на $\mathcal{A}_{\mathrm{D}}$ ). Ниже в теореме 3 будет установлен эпиморфизм из $\mathcal{A}_{\mathrm{D}}$ на $\mathcal{A}_{R}$, обратный к (16). Таким образом, утверждение теоремы доказано по модулю результата теоремы 3.

ПреДЛОЖениЕ 2. Пусть $\phi(x) \in \mathcal{A}_{\mathrm{Ch}}$ является образом $x \in \mathcal{A}_{R}$ под действием изоморфизма (16), тогда

$$
\phi\left(\Delta_{R}(x)\right)=\sigma \circ \Delta(\phi(x)) .
$$

ДокАЗАТЕЛЬСтво. Искомое утверждение может быть получено, применением $\pi_{z} \otimes \mathrm{id} \otimes \mathrm{id} \mathrm{к} \mathrm{соотношению}$

$$
\left(\mathrm{id} \otimes \Delta^{\mathrm{op}}\right) \mathcal{R}=\mathcal{R}_{12} \mathcal{R}_{13}
$$

которое эквивалентно второму равенству из (10).

3.4. От $R L L$-реализации к реализации Дринфельда. Сначала выразим все гауссовы образующие через $e_{1}^{ \pm}(z), f_{1}^{ \pm}(z), k_{1}^{ \pm}(z)$. Выражения

$$
\begin{aligned}
& k_{2}^{ \pm}(z)=k_{1}^{ \pm}(-q z) k_{1}^{ \pm}\left(q^{2} z\right)^{-1} \\
& k_{3}^{ \pm}(z)=k_{1}^{ \pm}\left(-q^{3} z\right)^{-1}
\end{aligned}
$$

немедленно следуют из (17). Соотношение (7) влечет

$$
\begin{aligned}
& \left(q^{2}-1\right) z\left(q^{3} z+w\right) L_{23}^{ \pm}(z) L_{33}^{ \pm}(w)+q(z-w)\left(q^{3} z+w\right) L_{33}^{ \pm}(z) L_{23}^{ \pm}(w)= \\
& =\left(q^{2} z-w\right)\left(q^{3} z+w\right) L_{23}^{ \pm}(w) L_{33}^{ \pm}(z),
\end{aligned}
$$


откуда

$$
q(z-w) k_{3}^{ \pm}(z) f_{2}^{ \pm}(w)=\left(q^{2} z-w\right) f_{2}^{ \pm}(w) k_{3}^{ \pm}(z)-\left(q^{2}-1\right) z f_{2}^{ \pm}(z) k_{3}^{ \pm}(z)
$$

Полагая $w=q^{2} z$, имеем

$$
f_{2}^{ \pm}\left(q^{2} z\right)=q^{-1} k_{3}^{ \pm}(z)^{-1} f_{2}^{ \pm}(z) k_{3}^{ \pm}(z)
$$

С другой стороны, соотношение (5) влечет

$$
f_{1}^{ \pm}(z)=-q^{-1 / 2} k_{3}^{ \pm}\left(-q^{-3} z\right)^{-1} f_{2}^{ \pm}\left(-q^{-3} z\right) k_{3}^{ \pm}\left(-q^{-3} z\right)
$$

Итак, мы получаем

$$
\begin{aligned}
& f_{2}^{ \pm}(z)=-q^{-1 / 2} f_{1}^{ \pm}(-q z), \\
& e_{2}^{ \pm}(z)=-q^{1 / 2} e_{1}^{ \pm}(-q z),
\end{aligned}
$$

где выражение для $e_{2}^{ \pm}(z)$ выводится аналогичным образом. Выражения для $e_{31}^{ \pm}(z)$ и $f_{13}^{ \pm}(z)$ будут получены в разделе 4 .

ЗАмЕЧАниЕ 3. Соотношения (19) можно также вывести непосредственно из явной формулы для $L^{ \pm}(z)$ аналогично тому, как мы проделали это для соотношений (17).

Теперь пусть

$$
\begin{aligned}
& E_{i}(z)=e_{i}^{+}(z)-e_{i}^{-}(z) \\
& F_{i}(z)=f_{i}^{+}(z)-f_{i}^{-}(z)
\end{aligned}
$$

тогда верны следующие равенства:

$$
\begin{aligned}
& E_{2}(z)=-q^{1 / 2} E_{1}(-q z), \\
& F_{2}(z)=-q^{-1 / 2} F_{1}(-q z) .
\end{aligned}
$$

Более того, из соотношений (4)-(7) следует

$$
\begin{gathered}
k_{1}^{ \pm}(z) k_{1}^{ \pm}(w)=k_{1}^{ \pm}(w) k_{1}^{ \pm}(z) \\
k_{1}^{-}(z) k_{1}^{+}(w)=k_{1}^{+}(w) k_{1}^{-}(z) ; \\
k_{1}^{ \pm}(z) E_{1}(w) k_{1}^{ \pm}(z)^{-1}=\frac{q(z-w)}{q^{2} z-w} E_{1}(w) \\
k_{1}^{ \pm}(z)^{-1} F_{1}(w) k_{1}^{ \pm}(z)=\frac{z-q^{2} w}{q(z-w)} F_{1}(w) \\
(q z+w)\left(z-q^{2} w\right) E_{1}(z) E_{1}(w)=(z+q w)\left(q^{2} z-w\right) E_{1}(w) E_{1}(z), \\
(z+q w)\left(q^{2} z-w\right) F_{1}(z) F_{1}(w)=(q z+w)\left(z-q^{2} w\right) F_{1}(w) F_{1}(z) ; \\
{\left[E_{1}(z), F_{1}(w)\right]=\left(q-q^{-1}\right) \delta\left(\frac{z}{w}\right)}
\end{gathered}
$$


Теорема 3. Изоморбизм ${ }^{4)}$ алгебр $\mathcal{A}_{\mathrm{D}}$ и $\mathcal{A}_{R}$ может быть установлен посредством следующих отображений:

$$
\begin{aligned}
q^{-1 / 4}\left(q-q^{-1}\right) e(q z) & \mapsto E_{1}(z), \\
q^{1 / 4}\left(q-q^{-1}\right) f(q z) & \mapsto F_{1}(z), \\
K^{ \pm}(q z) & \mapsto k_{1}^{ \pm}(z) k_{2}^{ \pm}(z)^{-1}
\end{aligned}
$$

ДокАЗАТЕЛЬство. Из формул (21) следует, что отображение (22) является гомоморфизмом. Для любого $n \geqslant 0$ гауссовы координаты $k_{1}^{ \pm}[n]$ можно шаг за шагом выразить из произведения $k_{1}^{ \pm}(z) k_{2}^{ \pm}(z)^{-1}$, откуда, учитывая соотношения (18), (20), получаем, что отображение (22) является эпиморфизмом. Наконец, можно проверить, что композиция отображений $(16),(13)$ и (22) является тождественным отображением.

\section{4. СВЯЗЬ СО СЛОЖНЫМИ ТОКАМИ}

В этом разделе мы напомним несколько определений, данных в работе [8], а затем выразим производящие функции $f_{13}^{ \pm}(z)$ и $e_{31}^{ \pm}(z)$ через токи Дринфельда.

Любая квантовая аффинная алгебра допускает два различных борелевских разложения. Борелевские подалгебры первого типа получаются из реализации Дринфельда. Пусть $U_{F}$ - подалгебра $U_{q}\left(A_{2}^{(2)}\right)$, порожденная $k^{ \pm 1}, f_{n}, n \in \mathbb{Z} ; a_{n}, n>0$, а $U_{E}$ - подалгебра $U_{q}\left(A_{2}^{(2)}\right)$, порожденная $k^{ \pm 1}, e_{n}, n \in \mathbb{Z} ; a_{n}, n<0$. "Токовая" борелевская подалгебра $U_{F}$ содержит подалгебру $U_{f}$, порожденную $f_{n}, n \in \mathbb{Z}$, а “токовая" борелевская подалгебра $U_{E}$ содержит подалгебру $U_{e}$, порожденную $e_{n}$, $n \in \mathbb{Z}$.

Борелевские подалгебры второго типа возникают из реализации Шевалле. Пусть $U_{q}\left(\mathfrak{b}_{+}\right)$и $U_{q}\left(\mathfrak{b}_{-}\right)$- подалгебры $U_{q}\left(A_{2}^{(2)}\right)$, порожденные соответственно $e_{\alpha}, e_{\delta-2 \alpha}, k_{\alpha}^{ \pm 1}$ и $e_{-\alpha}, e_{-(\delta-2 \alpha)}, k_{\alpha}^{ \pm 1}$. В образующих Дринфельда эти подалгебры порождены соответственно $k^{ \pm 1}, e_{0}, q f_{1} f_{0}-f_{0} f_{1}$ и $k^{ \pm 1}, f_{0}, q^{-1} e_{0} e_{-1}-e_{-1} e_{0}$.

Пусть $U_{f}^{-}, U_{F}^{+}, U_{e}^{+}$и $U_{E}^{-}$обозначают следующие пересечения борелевских подалгебр:

$$
\begin{array}{ll}
U_{f}^{-}=U_{F} \cap U_{q}\left(\mathfrak{b}_{-}\right), & U_{F}^{+}=U_{F} \cap U_{q}\left(\mathfrak{b}_{+}\right), \\
U_{e}^{+}=U_{E} \cap U_{q}\left(\mathfrak{b}_{+}\right), & U_{E}^{-}=U_{E} \cap U_{q}\left(\mathfrak{b}_{-}\right) .
\end{array}
$$

Верхний индекс отвечает той из борелевских подалгебр $U_{q}\left(\mathfrak{b}_{ \pm}\right)$, которая содержит рассматриваемую алгебру, а нижний индекс отвечает той “токовой” борелевской подалгебре $U_{F}$ или $U_{E}$, с которой берется пересечение. Эта буква прописная, если подалгебра содержит образующие, соответствующие мнимым корням, и строчная в противном случае.

Пусть $P^{+}$и $P^{-}$являются проекторами, определенными так, чтобы для любых $f_{+} \in U_{F}^{+}$и $f_{-} \in U_{f}^{-}$

$$
P^{+}\left(f_{-} f_{+}\right)=\varepsilon\left(f_{-}\right) f_{+}, \quad P^{-}\left(f_{-} f_{+}\right)=f_{-} \varepsilon\left(f_{+}\right) .
$$

\footnotetext{
4) Как и в замечании 2, описанное отображение является изоморфизмом ассоциативных алгебр. Оно сохраняет коединицу, но не сохраняет коумножения $\Delta$ и $\Delta_{\mathrm{D}}$.
} 
Пусть $P^{*+}$ и $P^{*-}$ также являются проекторами, определенными так, чтобы для любых $e_{+} \in U_{e}^{+}$и $e_{-} \in U_{E}^{-}$

$$
P^{*+}\left(e_{+} e_{-}\right)=e_{+} \varepsilon\left(e_{-}\right), \quad P^{*-}\left(e_{+} e_{-}\right)=\varepsilon\left(e_{+}\right) e_{-} .
$$

Наконец, определим сложные токи $s(z)$ и $r(z)$ следующим образом:

$$
\begin{aligned}
& s(z)=\left.\operatorname{res}\right|_{w=-q^{-1} z} f(z) f(w) \frac{d w}{w}, \\
& r(z)=\left.\operatorname{res}\right|_{w=-q z} e(w) e(z) \frac{d w}{w} .
\end{aligned}
$$

ЗАмЕчАниЕ 4. В работе [8] определялись токи $s(z)$ и $\tilde{s}(z)$. Определение $s(z)$ дословно совпадает с приведенным здесь, тогда как $\tilde{s}(z)=-s(-q z)$, или, что эквивалентно,

$$
\tilde{s}(z)=\left.\operatorname{res}\right|_{w=-q z} f(w) f(z) \frac{d w}{w} .
$$

В работе [8] также дано определение инволюции $\iota$ алгебры $U_{q}\left(A_{2}^{(2)}\right)$ :

$$
\iota\left(e_{n}\right)=f_{-n}, \quad \iota\left(f_{n}\right)=e_{-n}, \quad \iota\left(a_{n}\right)=a_{-n}, \quad \iota\left(K_{0}\right)=K_{0}^{-1} .
$$

Таким образом, ток $r(z)$ удовлетворяет равенству

$$
r(z)=\iota\left(-\tilde{s}\left(z^{-1}\right)\right) .
$$

ПрЕДЛОЖЕНИЕ 3. При изоморфизме (22) гауссовъ координаты $e_{31}^{ \pm}(z)$ u $f_{13}^{ \pm}(z)$ переходят в следующие выражения:

$$
\begin{aligned}
& e_{31}^{ \pm}(z) \mapsto \mp(1-q)\left(q^{-1}\left[P^{* \pm}\left(e\left(-q^{2} z\right)\right), e_{0}\right]_{q^{-1}}+\left[-q^{2} z e_{-1}, P^{* \pm}\left(e\left(-q^{2} z\right)\right) \mp e_{0}\right]_{q^{-1}}\right), \\
& f_{13}^{ \pm}(z) \mapsto \pm(1-q)\left(q\left[P^{ \pm}\left(f\left(-q^{2} z\right)\right), f_{0}\right]_{q^{-1}}+\left[\left(-q^{2} z\right)^{-1} f_{1}, P^{ \pm}\left(f\left(-q^{2} z\right)\right) \pm f_{0}\right]_{q^{-1}}\right) .
\end{aligned}
$$

ДокАзАТЕЛЬство. Мы рассматриваем лишь ток $f_{13}^{+}(z)$, так как для остальных токов рассуждения аналогичны. Соотношения (7) влекут

$$
\begin{aligned}
& \left(q^{2}-1\right) z\left(q^{3} z+w\right) l_{13}^{+}(z) l_{22}^{-}(w)+q(z-w)\left(q^{3} z+w\right) l_{23}^{+}(z) l_{12}^{-}(w)= \\
& \quad=\left(q^{2}-1\right) w\left(q^{3} z+w\right) l_{13}^{-}(w) l_{22}^{+}(z)+q(z-w)\left(q^{3} z+w\right) l_{12}^{-}(w) l_{23}^{+}(z) .
\end{aligned}
$$

Полагая $w=0$, имеем

$$
\left(q-q^{-1}\right) l_{13}^{+}(z)=\left[l_{12}^{-}(0), l_{23}^{+}(z)\right]
$$

или, что эквивалентно,

$$
\left(q-q^{-1}\right) f_{13}^{+}(z) k_{3}^{+}(z)=f_{1}^{-}[0] f_{2}^{+}(z) k_{3}^{+}(z)-f_{2}^{+}(z) k_{3}^{+}(z) f_{1}^{-}[0] .
$$

Еще раз используя (7), мы получаем

$$
k_{3}^{+}(z) f_{1}^{-}[0]=q f_{1}^{-}[0] k_{3}^{+}(z)+\left(q-q^{-1}\right) q^{1 / 2} f_{2}^{+}(z) k_{3}^{+}(z),
$$


откуда выводим

$$
f_{13}^{+}(z)=\frac{1}{q-q^{-1}}\left(q^{1 / 2} f_{1}^{+}(-q z) f_{1}^{-}[0]-q^{-1 / 2} f_{1}^{-}[0] f_{1}^{+}(-q z)\right)-q^{-1 / 2} f_{1}^{+}(-q z)^{2} .
$$

Теперь напомним один результат из работы [8]: с одной стороны, мы имеем

$$
\begin{aligned}
P\left(f\left(z_{1}\right) f\left(z_{2}\right)\right)=f^{+} & \left(z_{1}\right)\left(f^{+}\left(z_{2}\right)-\frac{q^{2}-1}{q^{2}-z_{2} / z_{1}} f^{+}\left(z_{1}\right)\right)- \\
& -\frac{\left(1+q^{3}\right)\left(1-z_{2} / z_{1}\right)}{(1+q)\left(q^{2}-z_{2} / z_{1}\right)\left(1+q z_{2} / z_{1}\right)} P\left(s\left(z_{1}\right)\right),
\end{aligned}
$$

а с другой -

$$
P\left(f\left(z_{1}\right) f\left(z_{2}\right)\right)=\frac{\left(q^{2}-z_{1} / z_{2}\right)\left(q^{-1}+z_{1} / z_{2}\right)}{\left(1-q^{2} z_{1} / z_{2}\right)\left(1+q^{-1} z_{1} / z_{2}\right)} P\left(f\left(z_{2}\right) f\left(z_{1}\right)\right) .
$$

Приравнивая правые части обоих соотношений, умножая равенство на $z_{2}$ и устремляя $z_{2}$ к бесконечности, мы получаем

$$
\begin{aligned}
\left(q-q^{-1}\right) P(f(z))^{2}= & \left(f_{1} P(f(z))-q^{-1} P(f(z)) f_{1}\right) z^{-1}+\frac{1+q^{3}}{q(1+q)} P(s(z))+ \\
& +\left(f_{1} f_{0}-q^{-1} f_{0} f_{1}\right) z^{-1}
\end{aligned}
$$

Наконец, так как изоморфизм (22) отображает $f_{1}^{ \pm}(-q z)$ в $\pm q^{1 / 4}\left(q-q^{-1}\right) P^{ \pm}\left(f\left(-q^{2} z\right)\right)$, мы выводим искомую формулу (25).

ТЕОрема 4. Изоморбизм (22) связывает гауссовъ координаты $f_{13}^{+}(z), e_{31}^{+}(z) u$ сложные токи $s(z), r(z)$ при помощи следующих соотношений:

$$
\begin{aligned}
P^{+}\left(s\left(-q^{2} z\right)\right) & =\frac{1}{\left(q-q^{-1}\right)\left(q-1+q^{-1}\right)} f_{13}^{+}(z), \\
P^{-}\left(s\left(-q^{2} z\right)\right) & =\frac{1}{\left(q-q^{-1}\right)\left(q-1+q^{-1}\right)}\left(f_{1}^{-}(z) f_{2}^{-}(z)-f_{13}^{-}(z)\right), \\
P^{*+}\left(r\left(-q^{2} z\right)\right) & =\frac{1}{\left(q-q^{-1}\right)\left(q-1+q^{-1}\right)} e_{31}^{+}(z), \\
P^{*-}\left(r\left(-q^{2} z\right)\right) & =\frac{1}{\left(q-q^{-1}\right)\left(q-1+q^{-1}\right)}\left(e_{2}^{-}(z) e_{1}^{-}(z)-e_{31}^{-}(z)\right) .
\end{aligned}
$$

ДокАЗАТЕльство. Для тока $s(z)$ утверждение немедленно следует из предложения 3 и теоремы 1 работы [8]. Для тока $r(z)$ придется также воспользоваться инволюцией $\iota$.

ЗАмечание 5 . Так как $f_{1}^{-}(z) f_{2}^{-}(z)-f_{13}^{-}(z)$ и $e_{2}^{-}(z) e_{1}^{-}(z)-e_{31}^{-}(z)$ являются в точности угловыми элементами гауссова разложения обратного $L$-оператора $\left(L^{-}(z)\right)^{-1}$, возможно, имеет смысл использовать элементы разложения именно обратного $L$-оператора $\left(L^{-}(z)\right)^{-1}$ в качестве гауссовых координат вместо элементов разложения обычного $L$-оператора $L^{-}(z)$. 
Благодарности. Автор благодарит С. М. Хорошкина за неоценимую помощь, многочисленные идеи и советы. Автор также благодарит С. З. Пакуляка за плодотворные обсуждения этой работы. Работа поддержана РФФИ (грант № 08-01-00667) и CNRS-PФФИ (грант № 09-01-93106-NCNIL) и Министерством образования и науки РФ (контракт 14.740.11.0081).

\section{Список литературы}

[1] A. Izergin, V. Korepin, Comm. Math. Phys., 79:3 (1981), 303-316.

[2] M. Jimbo, Comm. Math. Phys., 102:4 (1986), 537-547.

[3] В. О. Тарасов, ТMФ, 76:2 (1988), 184-198.

[4] D. Fioravanti, F. Ravanini, M. Stanishkov, Phys. Lett. B, 367:1-4 (1996), 113-120, arXiv: hep-th/9510047.

[5] S. M. Khoroshkin, V. N. Tolstoy, Lett. Math. Phys., 24:3 (1992), 231-244.

[6] V. Chari, A. Pressley, Comm. Math. Phys., 196:2 (1998), 461-476.

[7] J. Ding, S. M. Khoroshkin, Adv. Math., 189:2 (2004), 413-438.

[8] S. Khoroshkin, A. Shapiro, J. Geom. and Phys., 60:11 (2010), 1833-1851.

[9] В. Г. Дринфельд, Докл. АН СССР, 296:1 (1987), 13-17.

[10] Н. Ю. Решетихин, Л.А. Тахтаджян, Л.Д. Фаддеев, Алгебра и анализ, 1:1 (1989), 178-206.

[11] N. Yu. Reshetikhin, M. A. Semenov-Tian-Shansky, Lett. Math. Phys., 19:2 (1990), 133-142.

[12] J. T. Ding, I. B. Frenkel, Comm. Math. Phys., 156:2 (1993), 277-300.

[13] J. Ding, S. Khoroshkin, Lett. Math. Phys., 45:4 (1998), 331-352.

[14] W.-L. Yang, Y.-Z. Zhang, J. Phys. A, 34:13 (2001), L205-L211.

[15] D. Hernandez, Int. Math. Res. Not., 2010:1 (2010), 149-193, arXiv: 0704.2838.

[16] J. Ding, S. Khoroshkin, Transform. Groups, 5:1 (2000), 35-59.

[17] S. Khoroshkin, S. Pakuliak, J. Math. Kyoto Univ., 48:2 (2008), 277-321.

[18] B. Enriquez, S. Khoroshkin, S. Pakuliak, Comm. Math. Phys., 276:3 (2007), 691-725. 